\title{
Regulation of genomic imprinting by gametic and embryonic processes
}

\author{
J. Richard Chaillet, ${ }^{1}$ Debra S. Bader, and Philip Leder \\ Department of Genetics, Harvard Medical School, Howard Hughes Medical Institute, Boston, Massachusetts 02115 USA
}

\begin{abstract}
Parental genomic imprinting refers to the phenomenon by which alleles behave differently depending on the sex of the parent from which they are inherited. In the case of the murine transgene RSVIgmyc, imprinting is manifest in two ways: differential DNA methylation and differential expression. In inbred FVB/N mice, a transgene inherited from a male parent is undermethylated and expressed; a transgene inherited from the female parent is overmethylated and silent. Using a series of RSVIgmyc constructs and transgenic mice, we show that the imprinting of this transgene requires a cis-acting signal that is principally derived from the repeat sequences that make up the $3^{\prime}$ portion of the murine immunoglobulin $\alpha$ heavy-chain switch region. Such imprinting is relatively independent of the site of transgene insertion but is influenced by the structure of the transgene itself. Imprinting is also modulated by genetic background. Detailed studies indicate that the paternal allele is undermethylated and expressed in inbred $\mathrm{FVB} / \mathrm{N}$ mice and in heterozygous $\mathrm{F}_{1}$ $\mathrm{FVB} / \mathrm{N} / \mathrm{C57B1/6J}$ mice but is overmethylated and silent in inbred C57B1/6J mice. Consequently, the FVB/N genome appears to carry alleles of modulating genes that dominantly block methylation and permit expression of the paternally imprinted transgene. Furthermore, our results suggest that overmethylation is the default status of both parental alleles and that the paternal allele can be marked in trans by polymorphic factors that act in postblastocyst embryos.
\end{abstract}

[Key Words: Genomic imprinting; transgene; methylation; immunoglobulin; transcription]

Received February 24, 1995; revised version accepted April 4, 1995.

There is increasing evidence to indicate that apart from obvious sex-chromosome differences, the parental haploid contributions to the developing mammalian embryo are fundamentally unequal (McGrath and Solter 1984; Surani et al. 1986). This difference is manifest as a requirement of both maternal and paternal genomes for development to proceed normally. In nuclear transplanted mouse zygotes composed solely of maternal pronuclei (gynogenones) or solely of paternal pronuclei (androgenones|, there is a failure to complete embryogenesis. The genetic causes of this failure are localized to distinct chromosomal regions, identified by the association of phenotypic abnormalities with uniparental disomic inheritance of these regions (Searle and Beechey 1978; Cattanach and Kirk 1985).

These embryologic and genetic studies predicted the existence of distinct imprinted loci in which the two parental alleles are differentially marked (imprinted) in their respective germ lines. This prediction was realized with the identification of mouse transgenes in which the maternal allele was methylated differently than the paternal allele (Hadchouel et al. 1987; Reik et al. 1987; Sapienza et al. 1987; Swain et al. 1987). For the transgenic line TG.A, this methylation imprinting correlated

${ }^{1}$ Present address: Department of Biological Sciences, University of Pittsburgh, Pittsburgh, Pennsylvania 15260 USA. absolutely with expression imprinting, a difference in expression of the parental transgene alleles (Swain et al. 1987). More recently, a number of endogenous imprinted mouse genes have been identified, each with demonstrable expression differences between the maternal and the paternal alleles (Barlow et al. 1991; Bartolomei et al. 1991; DeChiara et al. 1991; Leff et al. 1992; Hatada et al. 1993; Giddings et al. 1994; Hayashizaki et al. 1994; Villar and Pedersen 1994; Guillemot et al. 1995). Interestingly, for at least four of these genes, associated methylation imprinting has also been demonstrated (Sasaki et al. 1992; Bartolomei et al. 1993; Ferguson-Smith et al. 1993; Hatada et al. 1993; Stoger et al. 1993; Hayashizaki et al. 1994|. The common feature of all of these loci is the distinction of the parental alleles at the molecular level, evident primarily as differences in which one of the parental alleles is silent, whereas the other is expressed.

For all imprinted loci, transgenic and endogenous, the underlying molecular imprinting mechanism is not known. Nonetheless, the study of imprinted transgenic mouse strains has provided insights into the role of developmental events (Chaillet et al. 1991; Ueda et al. 1992 \}, as well as the role of DNA methylation in this process (Chaillet 1992; Brandeis et al. 1993; Sasaki et al. 1993). One transgenic strain, TG.A, has been particularly informative (Swain et al. 1987; Chaillet et al. 1991). This line was created using the RSVIgmyc transgene that ex- 
presses c-myc derived from a murine Burkitt-like immunoglobulin heavy-chain $\alpha$ region/c-myc $(I g A / \mathrm{c}-m y c)$ translocation. The line dramatically exhibited both expression and methylation imprinting in which the relatively undermethylated, paternally derived allele is expressed in the heart (and no other tissue), whereas the overmethylated, maternally inherited allele is not expressed at all. The distinctive maternal pattern of overmethylation is established in the female gamete and maintained throughout adult life, whereas the distinctive paternal undermethylated pattern is established in the early embryo and maintained thereafter (Chaillet et al. 1991).

In the current work we have approached an understanding of the regulation of RSVIgmyc imprinting through a combination of molecular, genetic, and embryologic experiments. We first determined that multiple lines bearing the RSVIgmyc transgene and created in the $\mathrm{FVB} / \mathrm{N}$ inbred background all possess the same imprinting characteristics as the original TG.A line (Swain et al. 1987). Thus, the RSVIgmyc transgene is imprinted independently of the genomic integration site and maintains this characteristic in an inbred background (see below). Together, these observations indicate that the instructive sequences required for imprinting must be contained within the transgene. Furthermore, these transgenic mice should be contrasted with transgenic strains that appeared to be imprinted only on mixed strain backgrounds (Sapienza et al. 1989; Allen et al. 1990; Engler et al. 1991). In the latter cases, the apparent transgene parent-of-origin effect on methylation was the result of unlinked, strain-specific modifier alleles that were inherited in a parent-specific fashion (Allen and Mooslehner 1992; Sasaki et al. 1993).

We then assessed a series of mouse lines bearing systematically deleted transgene constructs. These enabled us to localize the cis-acting region necessary for transgene imprinting with respect to both methylation and expression. We also noted a difference in the penetrance of parental imprinting in two inbred mouse strains, $\mathrm{FVB} / \mathrm{N}$ and $\mathrm{C} 57 \mathrm{Bl} / 6$ ]. Developmental studies in appropriate homozygous and heterozygous mice of these strains allowed us to postulate the existence of transacting modulating factors that act in the early embryo to block a high level of paternal transgene methylation and thus permit its expression. Taken together, these results are consistent with a model in which a cis-acting signal attracts methylation to the maternal and paternal transgenes at different times in development, subject (in the paternal allele) to embryonic modification by the transacting products of genes that are present in at least two allelic forms in the mouse.

\section{Results}

Transgene imprinting is independent of integration site

Because of the potential effects of strain-specific modifier genes on the methylation of transgenes (Allen and Mooslehner 1992; Sasaki et al. 1993), we have continu- ally bred our transgene-bearing lines, TG.A and TG.B, into the inbred $\mathrm{FVB} / \mathrm{N}$ strain background where they display the imprinted phenotype, for example, undermethylation and expression of the paternal allele and overmethylation and silencing of the maternal allele (Chaillet et al. 1991). We have extended this analysis by examining four additional transgenic lines created directly in an $\mathrm{FVB} / \mathrm{N}$ strain background. As summarized in Table 1, all six lines (TG.A, TG.B, TG.C, TG.D, TG.E, and $T G . F \mid$ are parentally imprinted with respect to methylation and expression (when tested). For example, in the TG.C line (shown in Fig. 1A), the paternal allele is expressed in the heart, which is assayed as a 168-bp RNaseresistant, transgene-specific band. The maternal allele is transcriptionally silent. Using digestion with methylation-sensitive restriction enzymes as an assay of methylation state (Fig. 1B), it can be seen that the maternal allele is highly methylated (overmethylated), whereas the paternal allele is poorly methylated /undermethylated). Similar patterns of sex-of-parent-dependent methylation are also observed in the TG.D, TG.E, and TG.F lines. We therefore conclude that the transgene is a portable imprinted construct that is imprinted independently of the site of genomic integration and contains a cis-acting signal needed for expression and methylation imprinting.

\section{Identification of the cis-acting imprint signal}

We attempted to locate the cis-acting imprinting signal by analyzing a series of transgenic lines containing deletion constructs using expression and methylation assays.

Table 1. Imprinting characteristics of RSVIgmyc transgenic lines in an $F V B / N$ background

\begin{tabular}{lccrccc}
\hline & \multicolumn{2}{c}{ Allele methylation } & & \multicolumn{2}{c}{ Allele expression } \\
\cline { 2 - 3 } Line & maternal & paternal & & maternal & paternal \\
\hline TG.A & ++++ & + & + & - & + \\
TG.B $B^{a}$ & + & + & + & + \\
& ++++ & + & & + & + \\
TG.C & ++++ & + & & - & + \\
TG.D & ++++ & + & & N.T. & N.T. \\
TG.E & ++++ & + & & N.T. & N.T. \\
TG.F & ++++ & + & & N.T. & N.T. \\
\hline
\end{tabular}

The summary of the TG.A and TG.B lines is from Swain et al. (1987) and Chaillet et al. (1991). TG.C, TG.D, TG.E, and TG.F were created directly on an inbred FVB/N genetic background. Lines TG.D, TG.E, and TG.F were not examined for transgene expression. For methylation studies: ( $\mid$ The poorly methylated pattern in Fig. $1 B_{j}(++++)$ the highly methylated pattern in Fig. 1B. For expression studies, + refers to heart expression, and - refers to no detectable expression. (N.T.) Not tested.

${ }^{a}$ Of mice inherting a maternal transgene allele, $\sim 50 \%$ had a highly methylated, nonexpressing transgene phenotype, identical to the TG.A maternal allele. The remainder of the maternally inherited alleles were poorly methylated, transcriptionally active in the heart, and had a methylation pattern identical to the paternally transmitted TG.A allele (Chaillet et al. 1991). 


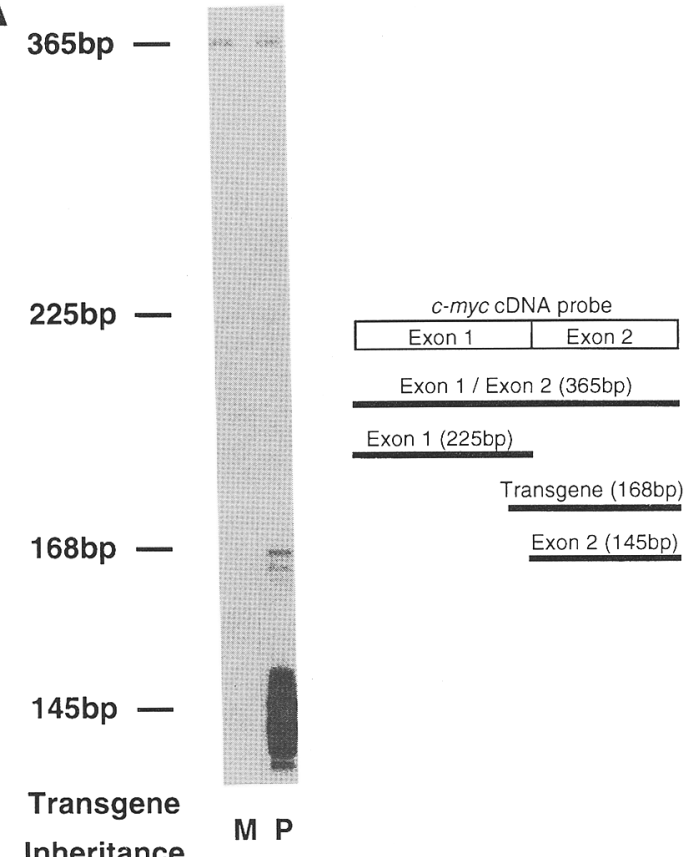

B

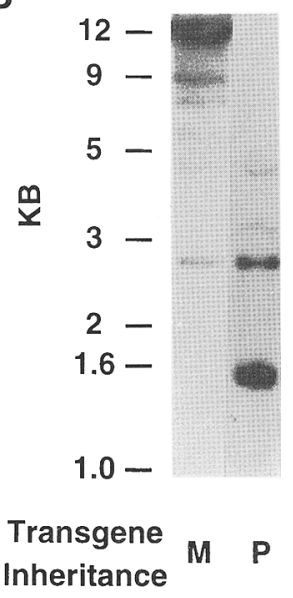

Figure 1. Genomic imprinting in an inbred strain background. The TG.C transgenic line was created directly in the inbred FVB/N strain. $(A)$ Ribonuclease protection assay showing TG.C expression of c-myc. Heart RNA was isolated from adult individuals of TG.C and analyzed for transgene-specific c-myc expression using a mouse c-myc cDNA fragment (365-bp NotI-PstI restriction fragment). The 168-bp band represents transcription of an $I g A / c-m y c$ fusion transcript containing the truncated transgene $\mathrm{c}-m y c$ exon $l(23$ $\mathrm{bp} /$ and intact exon 2 . Endogenous protected fragments of 225 and $365 \mathrm{bp}$ and a nonspecific (transgene or endogenous| fragment of $145 \mathrm{bp}$ are also evident. $(B)$ Southern blots showing methylation patterns of the parental alleles of TG.C. Tail DNA was isolated and digested with HpaII, and the Southern blot was hybridized with $\mathrm{C} \alpha .(\mathrm{M})$ Maternal; (P) paternal.
As can be seen in Figure $2 \mathrm{~A}$, the transgene is composed of three elements of different biological origins: $440 \mathrm{bp}$ of the Rous sarcoma virus long terminal repeat (RSV LTR), a $2.1-\mathrm{kb}$ portion of $\mathrm{pBR} 322$, and a $15-\mathrm{kb}$ EcoRI fragment containing the breakpoint region of a Burkitt-like translocation involving the mouse immunoglobulin $\alpha$ heavychain region $(\operatorname{Ig} A)$ and the c-myc proto-oncogene (Swain et al. 1987; Chaillet et al. 1991). This breakpoint region can also be conveniently divided into three sequence elements: a region comprising coding exons, intervening sequences, and switch repeat elements of $\operatorname{Ig} A(\mathrm{C} \alpha)$, relatively pure $\operatorname{Ig} A$ repetitive switch recombination sequences $(\mathrm{S} \alpha)$, and a truncated mouse c-myc gene. We arbitrarily define $\mathrm{C} \alpha$ as the 1756-bp EcoRI-XbaI restriction fragment between RSV and $S \alpha$ in the transgene construct (Fig. 2A). Germ-line $S \alpha$ consists of tandemly repeated homology units based on a primordial pentamer motif (Davis et al. 1980; Arakawa et al. 1993). It is important to note that our defined $\mathrm{C} \alpha$ region also contains copies of the switch repeat motif with homology decreasing with distance from S $\alpha$. Taking note of the above, we constructed a series of transgene deletions, each removing approximately one or more of these five distinct sequence elements. All lines were analyzed for methylation and, when appropriate, for expression. Because transcription of the transgene is initiated at a cryptic promoter site within the $\mathrm{S} \alpha$ region and because expression depends on the presence of enhancer sequences within the RSV element (D.S. Bader, unpubl.), removal of these elements precludes examination of expression but not methylation. Because c-myc is the reporter element, its removal also precludes examination of expression.

The imprinting characteristics of the transgene and several of the deletion lines are summarized in Figure $2 B$ and are shown in detail in the figures that follow. As would be expected, construct $C$, in which only the pBR322 sequences have been removed, maintains appropriate imprinted differences with respect to both methylation and expression (Fig. 3A,B). Construct $D$, deleted for $C \alpha$ sequences, also maintains both methylation and expression differences (Fig. 3A,B). Construct $B$, in which the RSV sequences have been deleted (and thus can only be assayed with respect to methylation), also preserves imprinting (Fig. 3B). Taken together, these analyses localize the required cis-acting imprinting signal to either the switch recombination sequences or $c-m y c$. Because these regions are required either for transcription of the transgene or for detection of its expression, deletion constructs that lack these regions can be assayed for methylation imprinting only.

Removal of major portions of the $c-m y c$ gene has no effect on methylation imprinting (construct $E$, Figs. 2 and 4). When further $c-m y c$ sequences and the majority of repetitive switch recombination sequences $\left(5^{\prime}\right.$ relative to the coding strand of the $\alpha$ gene) are removed, methylation imprinting also persists (construct $F$, Figs. 2 and 4). Furthermore, the $3^{\prime}$ myc sequences adjacent to pBR322 are not required because methylation imprinting is maintained despite their removal (construct $G$, Figs. 2 and 4). Only when a small (206 bp) region containing the junction of the switch recombination repeats and the $\mathrm{C} \alpha$ intron is deleted from construct $F$ (construct $H$, Figs. 2 and 4$)$ is methylation imprinting lost. This loss of imprinting is also observed in a more extensive deletion that includes this region (construct $I$, Figs. 2 and 4). In each of these cases, both parental alleles are highly and equally methylated, a situation also seen with the deletion of all mouse sequences, that is, pBR and RSV alone 


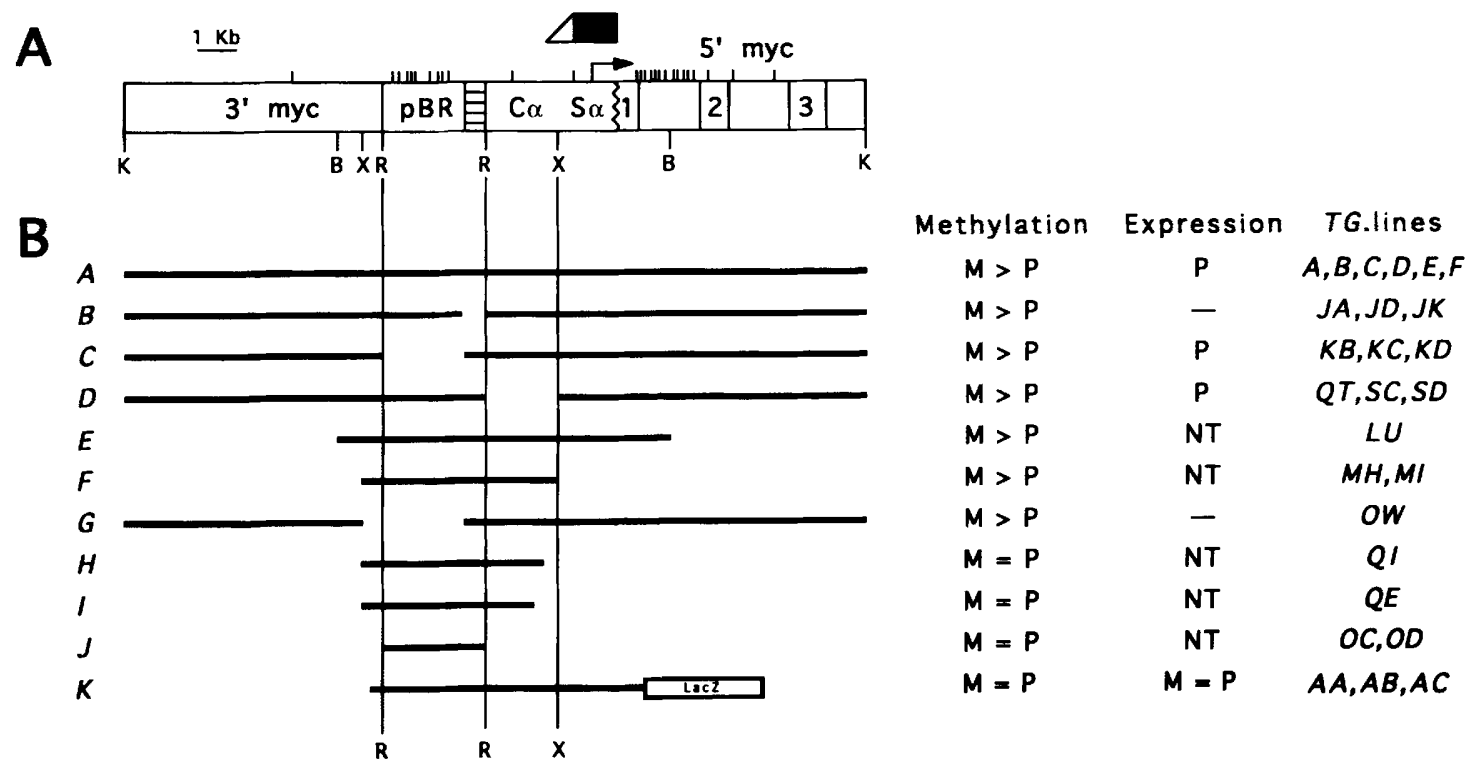

Figure 2. Summary of genomic imprinting of transgenic lines. All constructs are portrayed as the linearized versions injected into pronuclei of mouse zygotes. $(A)$ Physical characteristics of the transgene. MspI restriction endonuclease sites are shown as vertical marks at the top. The horizontally hatched area is the 440-bp PvuII-EcoRI fragment of the LTR of the Schmidt-Ruppin strain (subgroup D) of RSV (Yamamoto et al. 1980; Gorman et al. 1982). pBR is the EcoRI-AccI fragment of pBR322 containing Amp ${ }^{\mathrm{R}}$. The large EcoRI fragment of the circularized construct (bisected by the unique KpnI site) contains the breakpoint region of a Burkitt-like immunoglobulin $\alpha / \mathrm{c}-m y c$ translocation from the $\mathrm{S} 107$ plasmacytoma in which the 5 ' region of the switch recombination sequences of $\operatorname{IgA}(\mathrm{S} \alpha)$ has been translocated into the $5^{\prime}$ region of c-myc (Kirsch et al. 1981). Exon 1 of c-myc is truncated (and not shown to scale), leaving $23 \mathrm{bp}$ of the $3^{\prime}$ end intact (corresponds to bp 968-bp 990 of GenBank locus Muscmyc1). The arrow represents the approximate start of transcription in $\mathrm{S} \alpha$ of the $I g A / \mathrm{c}-m y c$ fusion transcript (D.S. Bader, unpubl.). C $\alpha$ is a 1756-bp EcoRI-XbaI fragment (with an internal $\mathrm{XbaI}$ sitc) containing intervening sequences and constant region coding exons (corresponds to bp 6374-bp 4620 of GenBank locus Musialpha, excluding internal sequence conflicts). Relevant methylation-sensitive BstUI sites (not shown) for the methylation patterns of constructs $C$ and $G$ are clustered in RSV (four sites) and the first intron of c-myc (eight sites). (1, 2, and 3) Exons 1-3 of mouse c-myc. Restriction sites: (B) BamHI; $\{\mathrm{X}\rangle \mathrm{XbaI} ;(\mathrm{R}) E c o \mathrm{RI} ;(\mathrm{K})$ KpnI. The density of the stippling and size of the bar above the IgA portion of the transgene correspond to the degree of homology of the repeated units, which decreases with distance from $S \alpha$. $|B|$ Imprinting characteristics of modified versions of the transgene. For construct $H$, the last 206 bp of C $\alpha$ has been deleted (corresponds to bp 4825-bp 4620 of Musialpha). For construct $l$, the last 355 bp of C $\alpha$ have been deleted (corresponds to bp 4973-bp 4620 of Musialpha). The transgenic lines created with these constructs are listed. Results are described as a difference or equivalence between maternal and paternal methylation or as expression in the heart. (-) No detectable expression from either maternal or paternal transgene alleles. (M) Maternal; (P) paternal; (NT) not tested.

(construct I, Figs. 2 and 4). Evidently, maternal-type overmethylation is the default methylation pattern.

\section{Is the endogenous IgA locus imprinted?}

Because a segment of the $I g A$ locus is required for transgene imprinting, it is logical to ask whether the $\operatorname{Ig} A$ locus itself is subject to parental imprinting. To test this possibility, it was necessary to detect an mRNA polymorphism between two inbred mouse strains so that products of each parental allele in an interspecific cross can be distinguished using an RNase protection assay. Fortunately, there are sequence polymorphisms in the 3 '-untranslated regions of immunoglobulin $\alpha$ heavychain mRNAs from inbred $\mathrm{C} 57 \mathrm{Bl} / 6 \mathrm{~J}$ and Mus spretus mice that allow us to distinguish them from each other. The results of an RNase protection experiment using splenic RNA derived from mice in which the C57Bl/6J and $M$. spretus alleles were reciprocally inherited from the male and female parents are shown in Figure 5. Very clearly, both alleles are expressed in the hybrid mice and the locus is evidently not subject to expression imprinting in this genetic background. Because the immunoglobulin loci undergo random allelic exclusion, this biallelic IgA expression must represent a mixture of monoallelically expressing cells (Alt et al. 1980). Consistent with the absence of expression imprinting, the $I g A$ locus also does not appear to be subject to methylation imprinting. Using a variety of methylation-sensitive restriction endonucleases, we were unable to detect methylation imprinting within the $\mathrm{C} \alpha$ and $\mathrm{S} \alpha$ sequences in the same interspecific crosses (data not shown).

\section{Penetrance of parental imprinting is influenced by genetic background}

In addition to the cis-acting requirement for genomic imprinting, trans-acting factors must also be involved in 
A

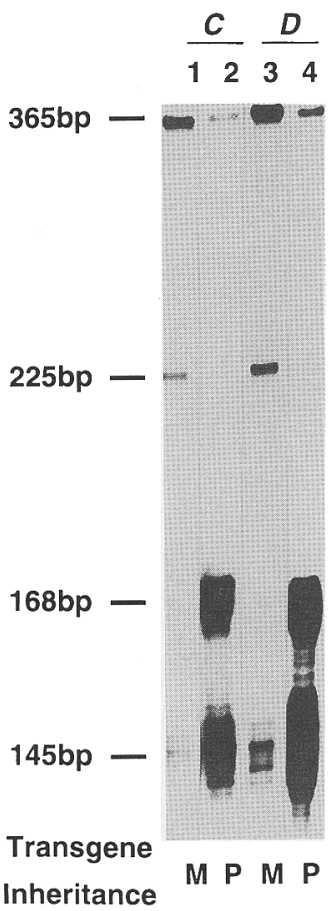

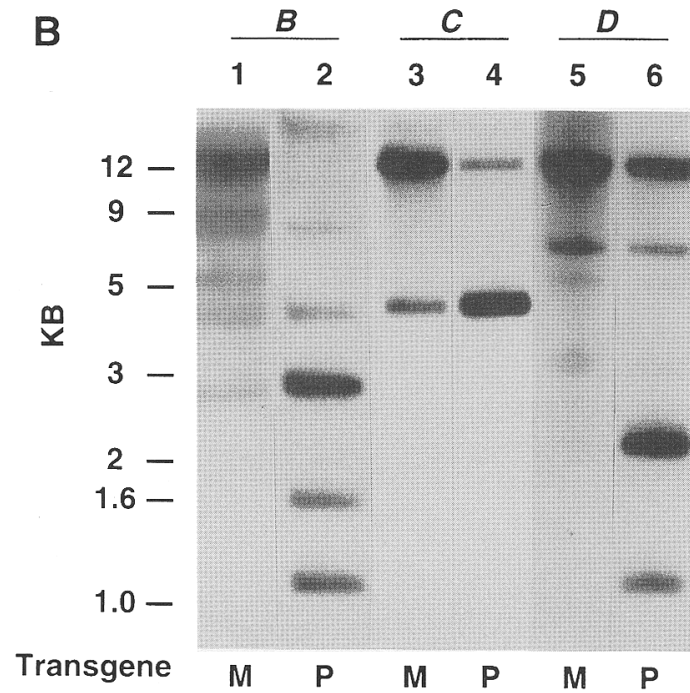

Inheritance
Figure 3. Imprinting analysis of transgene constructs. $\{A \mid$ Ribonuclease protection assay examining expression in transgenic lines TG.KD /created with construct $C$; lanes 1,2 ) and TG.QT (created with construct $D$, lanes 3,4$)$. The relationships of the probe to protected fragments are shown in Fig. 1A. (B) Southern blots of methylationsensitive endonuclease digests showing methylation patterns of imprinted transgenes. (Lanes 1,2) Alleles of TG.JD (created with construct $B\}$ digested with HpaII. (Lanes 3,4) Alleles of TG.KD (created with construct $C$ ) digested with BstUI. (Lanes 5,6 ) Alleles of TG.QT (created with construct $D$ ) digested with HpaII. All DNA samples were obtained from tails of adult mice and hybridized with either the probe $\mathrm{C} \alpha$ (TG.ID, TG.KD) or RSV (TG.QT). The parental source of the transgene is indicated at the bottom of each lane. creating an imprinted locus. In the case of our transgene, these factors presumably act directly or indirectly through the imprint signal that we have identified. The fact that imprinting of endogenous genes (Babinet et al. 1990; Latham and Solter 1991; Forejt and Gregorova 1992; Reik et al. 1993) and transgene methylation and expression (Engler et al. 1991; Allen et al. 1990) can be modified by strain background provided a genetic tool

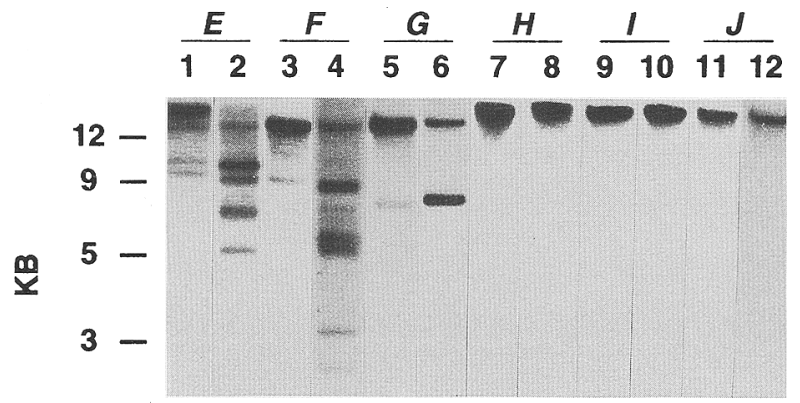

with which to study the putative trans-acting elements that influence parental imprinting. We noticed that as we passed our original imprinted transgene, TG.A (Swain et al. 1987; Chaillet et al. 1991), into the C57Bl/6J inbred strain, after eight generations, the paternally inherited transgene acquired the heavily methylated pattern normally associated with the maternally inherited allele (data not shown). These findings suggested that the paternal allele might be silenced in the inbred C57Bl/6J background.

To examine this possibility, we directly created a number of transgenic lines in an inbred C57Bl/6J back-

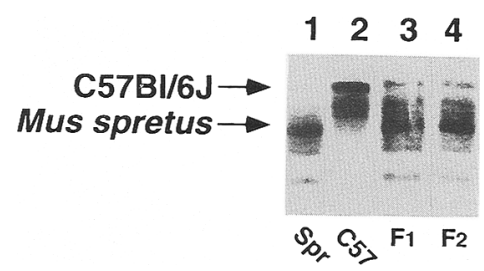

Figure 5. Biallelic expression of the endogenous $\lg A$ gene. Total spleen RNA was isolated from adult individuals and assayed for $I g A$ expression using a subcloned mouse $I g A$ genomic DNA fragment. The species-specific protected antisense fragments correspond to differences within the 3' UTR detected in a ribonuclease protection assay. (Lanes 1,2 ) Uniquely sized protected bands corresponding to $M$. spretus or C57Bl/6J alleles. (Lanes $3,4)$ Biallelic I $g A$ expression in interspecific crosses made between these species. (Lane 3) An $F_{1}$ hybrid between a C57Bl/6) female and a $M$. spretus male; (lane 4 ) a reciprocal cross to that shown in lane 3 such that the $M$. spretus allele is now maternally inherited. This $F_{2}$ animal was created by mating an $F_{1}$ female to a $\mathrm{C} 57 \mathrm{Bl} / 6$ J male. 
ground by using fertilized C57Bl/6J oocytes as targets for injection of the RSVIgmyc construct. One of these lines, TG.NL, demonstrated a complete loss of paternal allele expression that was associated with a high level of DNA methylation (Fig. 6A,B, lanes 1), a behavior that was identical to that of the maternal allele (Fig. 6A,B, lanes 2 ). If this aberrant paternal behavior is determined by modifier alleles present (or absent) in the $\mathrm{C} 57 \mathrm{Bl} / 6 \mathrm{~J}$ genome, movement of the transgene to the FVB/N background might restore the imprinted state. Accordingly, a wild-type FVB/N female was bred to a TG.NL (C57Bl/6J) male to produce $F_{1}$ offspring that inherit the transgene from their $\mathrm{C} 57 \mathrm{Bl} / 6 \mathrm{~J}$ father. As shown in Figure 6, A and $B$, lanes 3 , the paternally inherited transgene is transcriptionally active in the $F_{1}$ background and the transgene displays the characteristic undermethylated pattern associated with an imprinted paternal allele. Alternatively, in $\mathrm{F}_{1}$ offspring created by crossing a TG.NL (C57Bl/6J) female with a wild-type $\mathrm{FVB} / \mathrm{N}$ male, the maternal transgene remains silent and displays the overmethylated pattern of an imprinted maternal allele (Fig. 6A,B, lanes 4). These results indicate that the presence of the FVB/N haplogenome exerts a dominant effect that restores the activating imprint. Moreover, because in one cross the "silent" transgene is inherited from the C57Bl/6J father and activated by modifiying alleles provided in trans from the FVB/N mother, these factors must have established the paternal imprint by acting after fertilization. Because our earlier results established that the maternal overmethylated state is acquired during oogenesis and is maintained thereafter (Chaillet et al. 1991), the current results indicate that paternal imprinting occurs later, after zygote formation, and requires trans-acting factors, in this case, provided by the FVB/N genome.

\section{Paternal methylation pattern is acquired at a postblastocyst stage}

Because the paternal imprint is determined by events that take place after fertilization, we can begin to ask when during embryonic development this occurs. Formally, this question can only be answered in terms of transgene methylation as the expression of the transgene is limited to cardiac tissue and appears only after this organ forms (Swain et al. 1987; Jackson et al. 1990). Two observations indicate that paternal methylation occurs after the blastocyst stage of embryogenesis. First, previous developmental studies in FVB/N mice indicated that the paternal transgene is unmethylated in the blastocyst (Chaillet et al. 1991). The paternal transgene is also unmethylated in the C57Bl/6J blastocysts (Fig. 6B, lane 5), indicating that its adult methylation pattern is acquired after blastocyst formation. Second, founder FVB/N transgenic mice of the TG.C, TG.D, TG.E, and TG.F lines, created by injecting an unmethylated DNA construct into a fertilized oocyte, acquire adult methylation patterns identical to those characteristic of paternal imprinting (data not shown). Likewise, the transgenic founder (TG.NL) created in the $\mathrm{C} 57 \mathrm{Bl} / 6 \mathrm{~J}$ background by injecting an unmethylated construct displays an overmethylated pattern (Fig. 6B, lane 6) that must have been acquired during embryogenesis. These findings are con-
Figure 6. Examination of the imprinting characteristics of transgenic lines created on a $\mathrm{C} 57 \mathrm{Bl} / 6 \mathrm{~J}$ background. $(A)$ Ribonuclease protection assay examining transgene expression in the TG.NL C57Bl/6J transgenic line (lanes 1,2 ) and $F_{1}$ hybrids between TG.NL and wild-type FVB/N mice (lanes 3,4). (Lane 3) An $F_{1}$ hybrid between a wild-type $\mathrm{FVB} / \mathrm{N}$ female and a TG.NL male; (lane 4) an $\mathrm{F}_{1}$ hybrid between a TG.NL female and a wild-type FVB/N male. $(B)$ Southern blots of $H p a I I$ digests showing methylation patterns of the parental alleles of TG.NL mice (lanes 1,2,5,6,7) and $F_{1}$ hybrids between TG.NL and wild-type FVB/N mice (lanes 3,4). (Lane 3) An $F_{1}$ hybrid between a wild-type FVB/N female and a TG.NL male; (lane 4) an $\mathrm{F}_{1}$ hybrid between a TG.NL female and a wildtype FVB/N male; (lane 5) blastocyst DNA carrying the paternal allele of TG.NL. Thirty-nine blastocysts were obtained from matings between a $\mathrm{C} 57 \mathrm{Bl} / 6 \mathrm{~J}$ TG.NL male and wild-type C57Bl/6J females, and the DNA was extracted and digested with $\mathrm{HpaII}$ for this experiment. (Lane 6) The TG.NL founder transgenic mouse; (lane 7) day-7.5 TG.NL embryos containing a paternally derived transgene. Eight day-7.5 embryos from a mating between a TG.NL male and a wild-type $\mathrm{C} 57 \mathrm{Bl} / 6 \mathrm{~J}$ female were pooled, and the DNA was extracted and digested with $\mathrm{HpaII}$ for this experiment. All DNA samples except that of lanes 5 and 7 were derived from tails of adult mice. The Southern blots were hybridized with the C $\alpha$ probe.

A

$123 \quad 3$

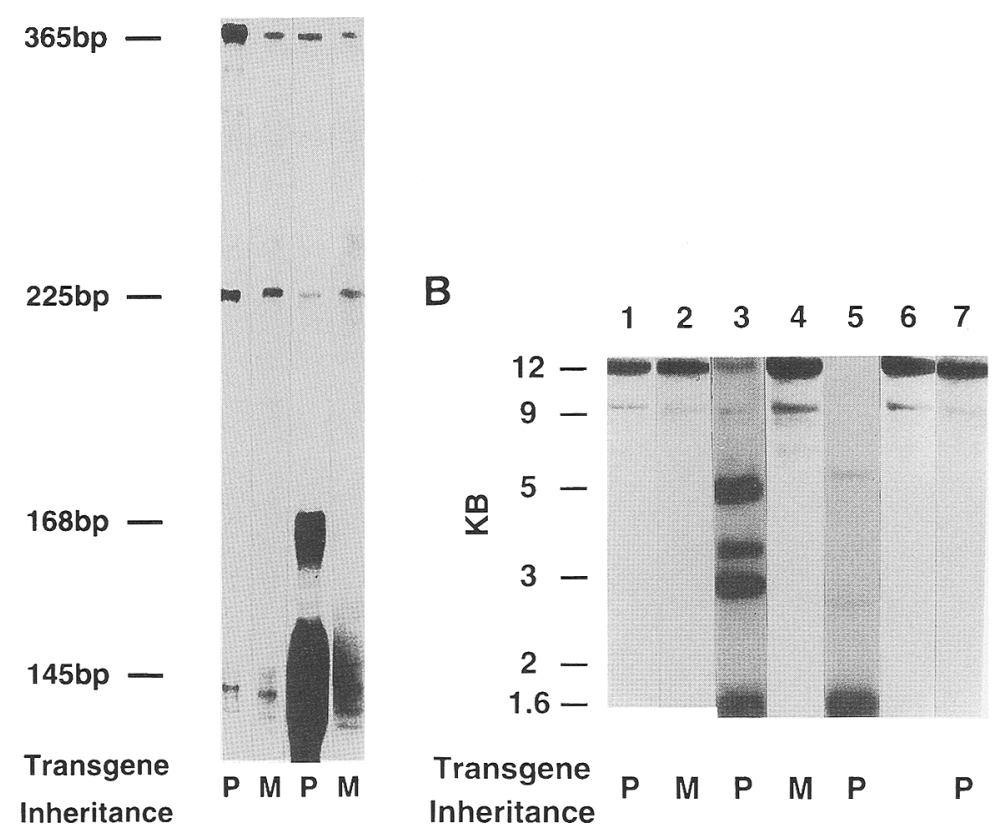


sistent with a highly methylated transgene in the adult arising from an unmethylated transgene in $\mathrm{C} 57 \mathrm{Bl} / 6 \mathrm{~J}$ blastocysts. Because day-7.5 C57Bl/6J embryos with a paternal TG.NL transgene exhibit the fully methylated pattern of the adult transgenic carrier (Fig. 6B, lane 7), the "adult" pattern must be acquired around the time of embryonic implantation. This timing coincides roughly with the onset of de novo methylation in the embryo (Monk et al. 1987).

\section{Discussion}

\section{The cis-acting imprint signal}

The fact that parental imprinting can be conveyed relatively independently of the genomic integration site by the RSVIgmyc transgene provides strong evidence that a cis-acting region within the transgene is necessary for its imprinted behavior. Using deletion analysis, we have systematically examined the transgene and identified within it a region that fulfills this requirement. The maximum size of the signal that is required to establish and/or maintain parental imprinting corresponds to our smallest loss-of-imprinting deletion, or $206 \mathrm{bp}$. It would be an oversimplification to assume that this region is sufficient to convey the imprint signal. Rather, it is subject to the influence of its immediate transgenic context and may require specific relationships to other sequences within the transgene. For example, we have shown that the endogenous $I g A$ locus of the mouse is not imprinted, although it carries this same 206-bp region. Obviously, the endogenous region resides in a very different immediate sequence context than does the transgenic copy. Furthermore, constructs in which the lac $Z$ gene is substituted for $\mathrm{c}-m y c$ do not display a methylation or expression imprint (construct $K$, Fig. 2B; data not shown), suggesting an interaction between separate elements of a more complex and more complete imprinting signal.

A number of mutation-associated imprinting phenomena in humans also emphasize the importance of subtle sequence relationships in genomic imprinting. Balanced germ-line translocations of chromosome 11 in the Beckwith-Weidemann syndrome (BWS) are always maternally derived (Pueschel and Padre-Mendoza 1984; Tommerup et al. 1993). This suggests that BWS translocations disrupt a normal imprinting mechanism or induce an abnormal mechanism. A recent report (Haas et al. 1992) suggests that the Philadelphia chromosome in patients with chronic myelogenous leukemia (CML) is derived from a balanced translocation of paternal chromosome 9 and maternal chromosome 22, creating a fusion of the $b c r$ and $a b l$ genes (Shtivelman et al. 1985; Grosveld et al. 1986). $b c r$ and $a b l$ are not likely to be imprinted in their germ-line configuration /Schwartzberg et al. 1991; Tybulewicz et al. 1991; Reik 1992; Melo et al. 1994; Riggins et al. 1994). Therefore, the somatically derived Philadelphia chromosomes in CML, if imprinted, must utilize "cryptic" germ-line imprint signals near bcr and/or $a b l$ to either direct the translocation or to select for an imprinted translocation in leukemic cells.
Interestingly, the region required for imprinting corresponds to the $3^{\prime}$ border of the $\operatorname{Ig} A$ switch recombination signal and contains the repeated primordial pentamers $[\mathrm{CTG}(\mathrm{A} / \mathrm{G}) \mathrm{G}]$ and related sequences that characterize this region. Repetitive DNA sequences are a recurrent theme among imprinted genes, and such sequences are associated with regions of allelic differences in DNA methylation (Neumann et al. 1995). For example, a 700bp cluster of direct repeats (with a 27-bp unit homology) are present in the mouse Igf2 gene, just upstream of the region where methylation differences between the parental alleles are apparent (Sasaki et al. 1992). Moreover, the differentially methylated region 2 of the imprinted Igf2/mpr gene (Stoger et al. 1993) also contains internally repeated DNA sequences. Taken together with the fact that repeated sequences can be regions of genetic instability and can influence chromosomal structure and, hence, gene expression [as in position effect variegation exhibited by arrays of repeated transgenes in Drosophila (Dorer and Henikoff 1994)], it is possible that repeated sequences in the appropriate context are a generic feature of imprint signals.

Finally, there is one example that draws together the joint themes of repeated sequences, recombination, and sensitivity to subtle alterations of gene structure. There is a strong parental inheritance preference in the fragile $\mathrm{X}$ syndrome (Laird 1987), a disorder associated with an expansion of a CGG trinucleotide repeat (Fu et al. 1991; Verkerk et al. 1991). At least an element of this preference may involve the induction of a maternal germ-line imprint. Such an imprint may in turn stimulate a series of postzygotic effects exemplified by trinucleotide repeat expansion, DNA methylation, and gene inactivation (Fu et al. 1991; Verkerk et al. 1991; Reyniers et al. 1993; Wohrle et al. 1993/. In view of the foregoing, it is possible that potentially imprintable sequences are abundant and that their properties are only realized when the appropriate sequence context has been created by subtle somatic recombinatorial events.

\section{Trans-acting control and timing of the imprinting mark}

We have already noted that trans-acting factors must be involved in creating an imprinted transgenic locus. Genetic evidence for this emerges from experiments in which we have shown that the imprinted properties of a transgene are lost in one genetic background (C57Bl/6J) but can be restored by breeding the transgene into another genetic background (FVB/N). Furthermore, because the imprint is restored in an $F_{1}$ hybrid formed between permissive and nonpermissive strains, we know that the responsible genetic modifiers act in a dominant fashion. Moreover, because the transgene can be donated by a male parent of the nonpermissive strain to be activated in an $F_{1}$ hybrid formed between that male and a female from the permissive strain, we conclude that the dominant modifier genes must act after fertilization.

The time and the molecular basis for marking the imprinted allele can be detailed further. Using methylation as our imprint marker, earlier results established that 
the (maternal) overmethylated state is acquired during oogenesis and is maintained thereafter (Chaillet et al. 1991). Our current results indicate that in contrast, the paternal allele does not acquire its distinctive methylation pattern until sometime after blastocyst formation but before day 7.5 of embryonic development. Furthermore, the distinctive paternal imprint occurs only in the presence of the products of the modifying genes supplied by the FVB/N genome. In its simplest form, this product would be a trans-acting factor supplied by the FVB/N genome that prevents hypermethylation of the paternal allele at the time of extensive embryonic de novo methylation (Monk et al. 1987).

It is worth mentioning that three other transgenic lines created directly in a $\mathrm{C} 57 \mathrm{Bl} / 6 \mathrm{~J}$ background displayed a somewhat weaker, variably imprinted phenotype (data not shown). Their paternal alleles were transcriptionally active at a level less than that observed in $\mathrm{FVB} / \mathrm{N}$, and their transgenes displayed a weakly maternal pattern of methylation. When these C57Bl/6J transgenic strains were bred with $\mathrm{FVB} / \mathrm{N}$, the $\mathrm{F}_{1}$ offspring displayed the strong methylation and expression phenotype characteristic of the $\mathrm{FVB} / \mathrm{N}$ background. Variation in imprinting behavior in the $\mathrm{C} 57 \mathrm{Bl} / 6 \mathrm{~J}$ background suggests that factors in addition to the strain-related modifying genes influence the extent of paternal de novo methylation during embryogenesis. It is possible that this property is influenced by the site or manner of genomic integration.

Endogenous imprinted loci also exhibit variability in genomic imprinting. For example, only the paternal allele of the Igf2 gene is normally active. However, in selected CNS regions (DeChiara et al. 1991) and certain tumors of somatic origin (Ogawa et al. 1993; Rainer et al. 1993), both parental alleles of $I g f 2$ are active and thus not imprinted. It is not difficult to imagine that this variability in Igf2 imprinting could develop in an analogous fashion to the methylation of both alleles of the RSVIgmyc transgene. The normally active paternal Igf2 allele is more methylated than the maternal allele. If the relatively poorly methylated maternal allele gains methylation in a directed (CNS) or sporadic (tumor) fashion following fertilization, it will become activated and result in biallelic Igf2 expression.

\section{The role of methylation}

Virtually every discussion of imprinting includes a consideration of the role that DNA methylation might play in establishing an imprinted pattern of gene expression. Early consideration of the RSVIgmyc transgene pointed out the clear correlation between the imprinted methylation and expression patterns (Swain et al. 1987; Chaillet et al. 1991). In support of this, strong correlations have also been observed between the levels of DNA methylation and transcription for endogenous imprinted genes (Sasaki et al. 1992; Bartolomei et al. 1993; FergusonSmith et al. 1993; Hatada et al. 1993; Stoger et al. 1993; Hayashizaki et al. 1994). The strongest evidence thus far comes from the observation that expression imprinting of three of these genes (Igf2, Igf2/mpr, and H19) is abolished on a cytosine methyltransferase-deficient mouse background, either because the normally silent allele is activated $(H 19)$ or because the normally active allele is silenced (Igf2 and Igf2/mpr) (Li et al. 1993).

The events we have discussed in terms of our imprinted transgene can be considered in the light of a model in which DNA methylation directly mediates one or more steps in the genomic imprinting process. The presence of different methylation patterns on the transgene in the mature male and female gametes establishes a chemical basis that allows one allele to be distinguished from the other. The highly methylated pattern of the oocyte-derived allele is propagated unchanged through subsequent stages of development. Because the paternal transgene is not highly methylated during spermatogenesis, it is unable to maintain its methylation through preimplantation embryogenesis. Subsequently, during postblastocyst embryogenesis, possibly using the system of methylation utilized by other imprinted genes (Li et al. 1993), the paternal allele acquires either a low level (FVB/N background) or a high level (C57Bl/6) background) of methylation. In such a methylation-dependent model, the maternal allele is silenced as a result of the highly methylated pattern it has acquired from the oocyte. The activity of the paternal allele, on the other hand, is regulated by the amount of embryonic de novo methylation acquired during the postblastocyst stage of embryogenesis, which depends on the trans-acting factors that are present.

\section{Materials and methods}

\section{Transgene DNA constructs}

DNA fragments injected into mouse zygotes for the creation of transgenic mice were either the original RSVIgmyc plasmid construct (Swain et al. 1987) or modified versions. Constructs containing pBR322 sequences were linearized prior to injection: for constructs $A, B$, and $D$ at the unique $K p n I$ site; for construct $E$ at the $B a m H I$ site of the circularized plasmid; for construct $F$ at the $X b a I$ site; and for construct / at the EcoRI site. Constructs with pBR322 sequences deleted (constructs $C$ and $G$ ) were constructed by placing plasmid vector sequences (pKS +, Stratagene) into the unique KpnI site of RSVIgmyc (Fig. 2). These vector sequences were removed by gel isolation prior to injection of the transgene fragment.

Constructs with partial deletions of $\mathrm{C} \alpha$ (constructs $H$ and $I$ ) were constructed using PCR and plasmid cloning techniques. The appropriate portion of $\mathrm{C} \alpha$ was constructed as an EcoRI-SalI PCR fragment to replace the entire $\mathrm{C} \alpha$ region of construct $F$. The XbaI-EcoRI 3' myc fragment was constructed as a SallEcoRI PCR fragment. The two PCR fragments were then cloned into the $\mathrm{pBR} / \mathrm{RSV}$ EcoRI vector, and the resultant plasmid was linearized with SalI prior to injection into fertilized oocytes.

For construct $K$, the bacterial lacZ gene has been substituted for c-myc, leaving $19 \mathrm{bp}$ of $5^{\prime} \mathrm{c}-m y c$ and $186 \mathrm{bp}$ of $3^{\prime} \mathrm{c}-\mathrm{myc}$ (defined by MspI and BglII sites near the $5^{\prime}$ and $3^{\prime}$ ends of the c-myc sequences, respectively). The lac $Z$ gene contains the SV40 nuclear transport signal, as well as a polyadenylation signal. 
Animals

Transgenic mice were created either in an inbred $\mathrm{FVB} / \mathrm{N}$ or in an inbred $\mathrm{C} 57 \mathrm{Bl} / 6 \mathrm{~J}$ genetic background. Founder transgenic mice were made by injection of the pronucleus of a fertilized egg (Palmiter and Brinster 1986). Transgenic lines were established from these founders, and the imprinting characteristics of the line were evaluated by passage through the maternal and paternal germ lines.

\section{Tissue and cell collection}

Transgene methylation was analyzed in blastocysts collected on day 3.5, postimplantation embryos on day 7.5 and adult tails. Blastocysts were collected from natural pregnancies on day 3.5 (day 0.5 is defined as the day that a vaginal plug was identified) by flushing the uterine horns and collecting the blastocysts free of contaminating maternal tissue (Hogan et al. 1986). Embryos from day 7.5 were collected by removal of the surrounding decidua and extraembryonic membranes.

\section{DNA analysis}

DNA from adult tails and embryos was isolated by ethanol precipitation after digestion of the tissue with proteinase $\mathrm{K}$ (Boehringer Mannheim, Indianapolis, IN), followed by phenolchloroform extraction. DNA was digested at the appropriate temperature with a 10 -fold excess $(\mathrm{U} / \mathrm{mg})$ of restriction endonuclease, electrophoresed on $1 \%$ agarose, and transferred to nylon filters (GeneScreen, DuPont) by the method of Southern (1975). The filters were hybridized at $42^{\circ} \mathrm{C}$ in $40 \%$ formamide and washed in $0.1 \times \mathrm{SSC}, 0.1 \% \mathrm{SDS}$, at $65^{\circ} \mathrm{C}$. The filters were hybridized with the probe $\mathrm{C} \alpha$, which is the 1756-bp EcoRI-XbaI fragment of $\mathrm{C} \alpha$. In constructs in which $\mathrm{C} \alpha$ was deleted, the 440-bp RSV fragment was used.

\section{RNA analysis}

Heart RNA was isolated by homogenizing tissue in a Polytron homogenizer (Brinkman Instruments, Westbury, NY) using a buffer containing $4 \mathrm{M}$ guanidine isothiocyanate, $2.5 \mathrm{~mm}$ sodium citrate ( $\mathrm{pH} 7$ ), and $0.1 \mathrm{M} \beta$-mercaptoethanol. Total RNA was isolated by the method of Chirgwin et al. (1979) by centrifugation through a $5.7 \mathrm{M}$ cesium chloride, $25 \mathrm{~mm}$ sodium acetate cushion. RNA was analyzed for transgene expression by ribonuclease protection assay (Melton et al. 1984). The probe used was a 365 -bp NotI-PstI fragment of the mouse c-myc cDNA that includes the $3^{\prime}$ portion of exon 1 and the $5^{\prime}$ portion of exon 2. Spleen RNA was isolated and analyzed for $I g A$ expression by ribonuclease protection assay (see above). The probe used to detect biallelic $\operatorname{Ig} A$ expression was a subcloned mouse $\operatorname{Ig} A$ genomic DNA fragment that includes the $3^{\prime}$ portion of the $I g A$ gene. This fragment is an $\sim 900$-bp EcoRI-HindIII restriction fragment that was cloned into pKS + (Stratagene) and linearized with ScaI.

\section{Acknowledgments}

We thank Ed Max for gifts of mouse $\operatorname{IgA}$ genomic clones and Judy Dunmore, Anne Harrington, and Steve Sansing for their technical support throughout this work. We are grateful to Argiris Efstratiadis, Howard Cedar, and Todd Evans for many helpful discussions of the manuscript.

The publication costs of this article were defrayed in part by payment of page charges. This article must therefore be hereby marked "advertisement" in accordance with 18 USC section 1734 solely to indicate this fact.

\section{References}

Allen, N.D. and K.A. Mooslehner. 1992. Imprinting, transgene methylation, and genotype-specific modification. Semin. Dev. Biol. 3: 87-98.

Allen, N.D., M.L. Norris, and M.A. Surani. 1990. Epigenetic control of transgene expression and imprinting by genotypespecific modifiers. Cell 61: 853-861.

Alt, F.W., V. Enea, A.L. Bothwell, and D. Baltimore. 1980. Activity of multiple light chain genes in murine myeloma cells producing a single functional light chain. Cell 21: 1-12.

Arakawa, H., T. Iwasato, H. Hayashida, A. Shimizu, T. Honjo, and H. Yamagishi. 1993. The complete murine immunoglobulin class switch region of the $\alpha$ heavy chain gene-hierarchic repetitive structure and recombination breakpoints. J. Biol. Chem. 268: 4651-4655.

Babinet, C., V. Richoux, J.-L. Guenet, and J.-P. Renard. 1990. The DDK inbred strain as a model for the study of intersection between parental genomes and egg cytoplasm in mouse preimplantation development. Development (Suppl.): 8188

Barlow, D.P., R. Stoger, B.G. Herrman, K. Saito, and N. Schweifer. 1991. The mouse insulin-like growth factor type-2 receptor is imprinted and closely linked to the Tme locus. Nature 349: 84-87.

Bartolomei, M.S., S. Zemel, and S.M. Tilghman. 1991. Parental imprinting of the mouse $\mathrm{Hl} 9$ gene. Nature 351: 153-155.

Bartolomei, M.S., A.L. Webber, M.E. Brunkow, and S.M. Tilghman. 1993. Epigenetic mechanisms underlying the imprinting of the mouse $H 19$ gene. Genes \& Dev. 7: 1663-1667.

Brandeis, M., T. Kafri, M. Ariel, J.R. Chaillet, J. McCarrey, A. Razin, and H. Cedar. 1993. The ontogeny of allele-specific methylation associated with imprinted genes in the mouse. EMBO I. 12: 3669-3677.

Cattanach, B.M. and M. Kirk. 1985. Differential activity of maternally and paternally derived chromosomal regions in mice. Nature 315: 496-498.

Chaillet, J.R. 1992. DNA methylation and genomic imprinting in the mouse. Semin. Dev. Biol. 3: 99-105.

Chaillet, J.R., T.F. Vogt, D.R. Beier, and P. Leder. 1991. Parentalspecific methylation of an imprinted transgene is established during gametogenesis and progressively changes during embryogenesis. Cell 66: 77-83.

Chirgwin, J.M., A.E. Przybyla, R.J. McDonald, and W.J. Rutter. 1979. Isolation of biologically active ribonucleic acid from sources enriched in ribonuclease. Biochemistry 18: 5294 5299.

Davis, M.M., S.K. Kim, and L.E. Hood. 1980. DNA sequences mediating class switching in alpha-immunoglobulins. Science 209: 1360-1365.

DeChiara, T.M., E.J. Robertson, and A. Efstratiadis. 1991. Parental imprinting of the mouse insulin-like growth factor II gene. Cell 64: 849-859.

Dorer, D.R. and S. Henikoff. 1994. Expansions of transgene repeats cause heterochromatin formation and gene silencing in Drosophila. Cell 77: 993-1002.

Engler, P., D. Haasch, C.A. Pinkert, L. Doglio, M. Glymour, R. Brinster, and U. Storb. 1991. A strain-specific modifier on mouse chromosome 4 controls the methylation of independent transgene loci. Cell 65: 939-947.

Ferguson-Smith, A.C., H. Sasaki, B.M. Cattanach, and M.A. Surani. 1993. Parental-origin-specific epigenetic modifications 
of the mouse H19 gene. Nature 362: 751-755.

Forejt, J. and S. Gregorova. 1992. Genetic analysis of genomic imprinting: An imprintor-1 gene controls inactivation of the paternal copy of the mouse Tme locus. Cell 70: 443-450.

Fu, Y.H., D.P.A. Kuhl, A. Pizzuti, M. Pieretti, J.S. Sutcliffe, S. Richards, A.J.M.H. Verkerk, J.J.A. Holden, R.G. Fenwick, and S.T. Warren. 1991. Variation of the CGG repeat at the fragile $X$ site results in genetic instability: Resolution of the Sherman paradox. Cell 67: 1047-1058.

Giddings, S.J., C.D. King, K.W. Harman, J.F. Flood, and L.R. Carnaghi. 1994. Allele-specific inactivation of insulin 1 and 2 in the mouse yolk sac indicates imprinting. Nature Genet. 6: $310-313$.

Gorman, C.M., G.T. Merlino, M.C. Willingham, I. Pastan, and B.H. Howard. 1982. The Rous sarcoma virus long terminal repeat is a strong promoter when introduced into a variety of eukaryotic cells by DNA-mediated transfection. Proc. Natl. Acad. Sci. 79: 6777-6781.

Grosveld, G.T., T. Verwoerd, T. van Agthoven, A. deKlein, K.L. Ramachandran, N. Heisterkamp, K. Stam, and J. Grotten. 1986. The chronic myelocytic cell line K562 contains a breakpoint in $b c r$ and produces a chimeric $b c r / a b l$ transcript. Mol. Cell. Biol. 6: 607-616.

Guillemot, F., T. Caspary, S.M. Tilghman, N.G. Copeland, D.J. Gilbert, N.A. Jenkins, D.J. Anderson, A.L. Joyner, J. Rossant, and A. Nagy. 1995. Genomic imprinting of Mash2, a mouse gene required for trophoblast development. Nature Genet. 9: $235-242$.

Haas, O.A., A. Argyriou-Tirita, and T. Lion. 1992. Parental origin of chromosomes involved in the translocation $t(9 ; 22)$. Nature 359 414-416.

Hadchouel, M., H. Farza, D. Simon, P. Tiollais, and C. Pourcel. 1987. Maternal inhibition of hepatitis B surface antigen gene expression in transgenic mice correlates with de novo methylation. Nature 329: 454-456.

Hatada, I., T. Sugama, and T. Mukai. 1993. A new imprinted gene cloned by a methylation-sensitive genome scanning method. Nucleic Acids Res. 21: 5577-5582.

Hayashizaki, Y., H. Shibata, S. Hirotsune, H. Sugino, Y. Okazaki, N. Sasaki, K. Hirose, H. Inoto, H. Okuizumi, M. Muramatsu, H. Komatsubara, T. Shiroishi, K. Moriwaki, M. Katsuki, N. Hatano, H. Sasaki, T. Ueda, N. Mise, N. Takagi, C. Plass, and V.M. Chapman. 1994. Identification of an imprinted U2af binding protein related sequence on mouse chromosome 11 using the RLGS method. Nature Genet. 6: $33-40$.

Hogan, B., F. Costantini, and E. Lacy. 1986. Manipulating the mouse embryo. Cold Spring Harbor Laboratory, Cold Spring Harbor, New York.

Jackson, T., M.F. Allard, C.M. Sreenan, L.K. Doss, S.P. Bishop, and J.L. Swain. 1990. The $c-m y c$ proto-oncogene regulates cardiac development in transgenic mice. Mol. Cell. Biol. 10: 3709-3716.

Kirsch, I.R., J.V. Ravetch, S.P. Kwan, E.E. Max, R.L. Ney, and P. Leder. 1981. Multiple immunoglobulin switch region homologies outside the heavy chain constant region locus. $\mathrm{Na}$ ture 293: 585-587.

Laird, C.D. 1987. Proposed mechanism of inheritance and expression of the human fragile- $X$ syndrome of mental retardation. Genetics 117: 587-599.

Latham, K.E. and D. Solter. 1991. Effect of egg composition on the developmental capacity of androgenetic mouse embryos. Development 113: 561-568.

Leff, S.E., C.I. Brannan, M.L. Reed, T. Ozcelik, U. Franke, N.G. Copeland, and N.A. Jenkins. 1992. Maternal imprinting of the mouse Snrpn gene and conserved linkage homology with the human Prader-Willi syndrome region. Nature Genet. 2: 259-264.

Li, E., C. Beard, and R. Jaenisch. 1993. Role for DNA methylation in genomic imprinting. Nature 366: 362-365.

McGrath, J. and D. Solter. 1984. Completion of mouse embryogenesis requires both the maternal and paternal genomes. Cell 37: 179-183.

Melo, J.V., X.-H. Yan, J. Diamond, and J.M. Goldman. 1994. Lack of imprinting of the $A B L$ gene. Nature Genet. 8: 318 319.

Melton, D.A., P.A. Krieg, M.R. Rebagliati, T. Maniatis, K. Zinn, and M.R. Green. 1984. Efficient in vitro synthesis of biologically active RNA and RNA hybridization probes from plasmids containing a bacteriophage SP6 promoter. Nucleic Acids Res. 12: 7035-7056.

Monk, M., M. Boubelik, and S. Lehnert. 1987. Temporal and regional changes in DNA methylation in the embryonic, extraembryonic and germ cell lineages during mouse embryo development. Development 99: 371-382.

Neumann, B., P. Kubicka, and D.P. Barlow. 1995. Characteristics of imprinted genes. Nature Genet. 9: 12-13.

Ogawa, O., M.R. Eccles, J. Szeto, L.A. McNoe, K. Yun, M.A. Mow, P.J. Smith, and A.E. Reeve. 1993. Relaxation of insulin-like growth factor gene imprinting implicated in Wilms' tumour. Nature 362: 749-751.

Palmiter, R.D. and R.L. Brinster. 1986. Germ-line transformation of mice. Annu. Rev. Genet. 20: 465-499.

Pueschel, S.M. and T. Padre-Mendoza. 1984. Chromosome 11 and the Beckwith-Weidemann syndrome. I. Pediatr. 104: 484-485.

Rainier, S., L.A. Johnson, C.J. Dobry, A.J. Ping, P.E. Grundy, and A.P. Feinberg. 1993. Relaxation of imprinted genes in human cancer. Nature 362: 747-749.

Reik, W. 1992. Imprinting in leukaemia. Nature 359: 362-363.

Reik, W., A. Collick, M.L. Norris, S.C. Barton, and M.A. Surani. 1987. Genomic imprinting determines methylation of parental alleles in transgenic mice. Nature 328: 248-251.

Reik, W., I. Romer, S.C. Barton, M.A. Surani, S.K. Howlett, and J. Klose. 1993. Adult phenotypes in the mouse can be affected by epigenetic events in the early embryo. Development 119: 933-942.

Reyniers, E., L. Vits, K. De Boulle, B. Van Roy, D. Van Velzen, E. de Graaff, A.J.M.H. Verkerk, H.J.Z. Jorens, J.K. Darby, B. Oostra, and P. Willems. 1993. The full mutation in the FMR-1 gene of male fragile $X$ patients is absent in their sperm. Nature Genet. 4: 143-146.

Riggins, G.J., F. Zhang, and S. Warren. 1994. Lack of imprinting of BCR. Nature Genet. 6: 226.

Sapienza, C., A.C. Peterson, J. Rossant, and R. Balling. 1987. Degree of methylation of transgenes is dependent on gamete of origin. Nature 328: 251-254.

Sapienza, C., J. Paquette, T.H. Tran, and A. Peterson. 1989. Epigenetic and genetic factors affect transgene methylation imprinting. Development 107: 165-168.

Sasaki, H., P.A. Jones, J.R. Chaillet, A.C. Ferguson-Smith, S.C. Barton, W. Reik, and M.A. Surani. 1992. Parental imprinting: Potentially active chromatin of the repressed maternal allele of the mouse insulin-like growth factor II (Igf2) gene. Genes \& Dev. 6: 1843-1856.

Sasaki, H., N.D. Allen, and M.A. Surani. 1993. DNA methylation and genomic imprinting in mammals. In DNA methylation: Molecular biology and biological significance (ed. J.P. Jost and H.P. Saluz|, pp. 469-486. Birkhauser Verlag, Basel, Switzerland.

Schwartzberg, P.L., A.M. Stall, J.D. Hardin, K.S. Bowdish, T. Humaran, S. Boast, M.L. Harbison, E.J. Robertson, and S.P. 
Goff. 1991. Mice homozygous for the $a b 1^{\mathrm{ml}}$ mutation show poor viability and depletion of selected $B$ and $T$ cell populations. Cell 65: 1165-1175.

Searle, A.G. and C.V. Beechey. 1978. Complementation studies with mouse translocations. Cytogenet. Cell Genet. 20: 282 303.

Shtivelman, E., B. Lifshitz, R.P. Gale, and E. Canaani. 1985. Fused transcript of $a b l$ and $b c r$ genes in chronic myelogenous leukaemia. Nature 315: 550-554.

Southern, E.M. 1975. Detection of specific sequences among DNA fragments separated by gel electrophoresis. $J$. Mol. Biol. 98: 503-517.

Stoger, R., P. Kubicka, C.-G. Liu, T. Kafri, A. Razin, H. Cedar, and D. P. Barlow. 1993. Maternal-specific methylation of the imprinted mouse Igf2r locus identifies the expressed locus as carrying the imprinting signal. Cell 73: 61-71.

Surani, M.A.H., S.C. Barton, and M.L. Norris. 1986. Nuclear transplantation in the mouse: Heritable differences between parental genomes after activation of the embryonic genome. Cell 45: 127-136.

Swain, J.L., T.A. Stewart, and P. Leder. 1987. Parental legacy determines methylation and expression of an autosomal transgene: A molecular mechanism for parental imprinting. Cell 50: 719-727.

Tommerup, N., C.A. Brandt, S. Pedersen, L. Bolund, and J. Kamper. 1993. Sex dependent transmission of Beckwith-Weidemann syndrome associated with a reciprocal translocation t(9:11)(pl1.2;p15.5). I. Med. Genet. 30: 958-961.

Tybulewicz, V.L.J., C.E. Crawford, P.K. Jackson, R.T. Bronson, and R.C. Mulligan. 1991. Neonatal lethality and lymphopenia in mice with a homozygous disruption of the $c$ - $a b l$ protooncogene. Cell 65: 1153-1163.

Ueda, T., K. Yamazaki, R. Suzuki, H. Fuijimoto, H. Sasaki, Y. Sakaki, and T. Higahinakagawa. 1992. Parental methylation patterns of a transgenic locus in adult somatic tissues are imprinted during gametogenesis. Development 116: 831839.

Verkerk, A.J.M.H., M. Pieretti, J.S. Sutcliffe, Y.-H. Fu, D.P.A. Kuhl, A. Pizzuti, O. Reiner, S. Richards, M.F. Victoria, F. Zhang, B.E. Eussen, G.-J.B. van Ommen, L.A.J. Blonden, G.J. Riggins, J.L. Chastain, C.B. Kunst, H. Galjaard, C.T. Caskey, D.L. Nelson, B.A. Oostra, and S. Warren. 1991. Identification of a gene (FMR-1) containing a CGG repeat coincident with a breakpoint cluster region exhibiting length variation in Fragile X syndrome. Cell 65: 905-914.

Villar, A.J. and R.A. Pedersen. 1994. Parental imprinting of the Mas protooncogene in mouse. Nature Genet. 8: 373-379.

Wohrle, D., I. Hennig, W. Vogel, and P. Steinbach. 1993. Mitotic stability of Fragile X mutations in differentiated cells indicates early post-conceptional trinucleotide repeat expansion. Nature Genet. 4: 140-142.

Yamamoto, T., G. Jay, and I. Pastan. 1980. Unusual features in the nucleotide sequence of a cDNA clone derived from the common region of avian sarcoma virus messenger RNA. Proc. Natl. Acad. Sci. 77: 176-180. 


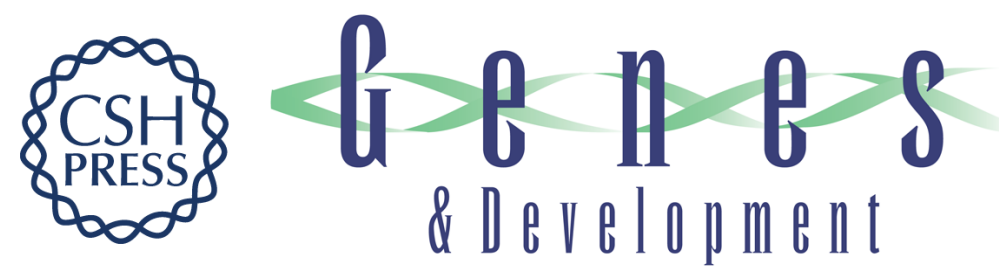

\section{Regulation of genomic imprinting by gametic and embryonic processes.}

J R Chaillet, D S Bader and P Leder

Genes Dev. 1995, 9:

Access the most recent version at doi:10.1101/gad.9.10.1177

References This article cites 64 articles, 15 of which can be accessed free at:

http://genesdev.cshlp.org/content/9/10/1177.full.html\#ref-list-1

License

Email Alerting

Service

Receive free email alerts when new articles cite this article - sign up in the box at the top right corner of the article or click here.

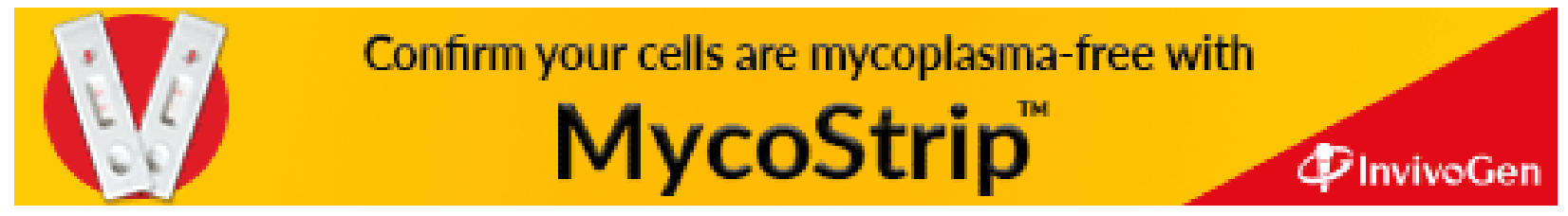

